Innovaciones de Negocios, 13(26): 299 - 316

(c) 2016 UANL, Impreso en México (ISSN 2007-1191)

Fecha de recepción: 08 de marzo de 2016. Fecha de aceptación: 27 de octubre de 2016.

\title{
Visión empresarial a través del espíritu emprendedor de los estudiantes que cursan la unidad de aprendizaje del ámbito empresarial en la UANL, FACPYA (Business vision through its entrepreneurial spirit of the students who attend the Learning Unit of the business sector in the UANL, FACPYA)
}

\author{
Ma. Margarita Carrera Sánchez \\ Abel Partida Puente* \\ Luis A. Villarreal Villarreal*
}

\begin{abstract}
This research aims to recognize the characteristics that form the entrepreneurship and how these essential characteristics contribute to the entrepreneurial vision of university students of the Facultad de Contaduría Pública y Administración of UANL in the development of new businesses, identifying the attributes that foster entrepreneurship, such as: the ability to face risk situations, the tools, the knowledge, the values, the abilities, the entrepreneurial profile and to demonstrate how those attributes affect the development of college students in the creation of new businesses. The collected data was obtained from a questionnaire, validated and reliable; it was examined with the statistical package SPSS-V18. Using multiple linear regression, a goodness-of-fit test of the normal distribution, heterogeneity and linearity was performed. The results show the significance of the variable (X4), the values and personality traits $(X 6)$, with a positive impact on college students in generating new business, while the study variables (X1) skills, (X2) tools, (X3) knowledge, (X5) talents, are not significant. The study was delimited to students of the Universidad Autónoma de Nuevo Leon, the scientific method was used through a quantitative and qualitative study based on a simple linear regression.
\end{abstract}

Keywords: abilities, business vision, entrepreneurship, knowledge, personality traits, values

JEL: M13, M53

\footnotetext{
- Universidad Autónoma de Nuevo León, Facultad de Contaduría Pública y Administración, San Nicolás de los Garza, N.L., México. Email: magaly_carrera@hotmail.com

* Universidad Autónoma de Nuevo León, Facultad de Contaduría Pública y Administración, San Nicolás de los Garza, N.L., México. Email: abelpartida@hotmail.com

- Universidad Autónoma de Nuevo León, Facultad de Contaduría Pública y Administración, Centro de Desarrollo Empresarial y Posgrado, San Nicolás de los Garza, N.L., México. Email: Ivillarreal@yahoo.com
} 
Resumen: La presente investigación, tiene como finalidad conocer las características que configuran el espíritu emprendedor y de qué manera contribuyen estas características esenciales en la visión empresarial de los estudiantes universitarios de la Facultad de Contaduría Pública y Administración de la UANL, en el desarrollo de nuevos negocios, identificando los atributos que propician el espíritu emprendedor como son: las habilidades para enfrentar situaciones de riesgo, las herramientas, los conocimientos, los valores, los talentos y los rasgos de personalidad y demostrar en qué medida inciden en el desarrollo de los estudiantes universitarios para la creación de nuevos negocios. Los datos recolectados se obtuvieron de un cuestionario con su prueba de validez y confiabilidad, se examinaron con el paquete estadístico SPSS-V18. Mediante una regresión lineal múltiple, se realizó la prueba de bondad de ajuste de la distribución normal, la heterogeneidad y la linealidad. Los resultados muestran la significancia de la variable $\left(\mathrm{X}_{4}\right)$ valores y los rasgos de personalidad $\left(X_{6}\right)$, con un impacto positivo en los estudiantes universitarios en la generación de nuevos negocios, mientras que las variables de estudio $\left(X_{1}\right)$ habilidades, $\left(X_{2}\right)$ las herramientas, $\left(X_{3}\right)$ los conocimientos, $\left(X_{5}\right)$ los talentos, no son significativas. El estudio se delimito a estudiantes de la Universidad Autónoma de Nuevo León, se utilizó el método científico mediante un estudio cuantitativo y cualitativo de acuerdo a una regresión lineal simple

Palabras clave: conocimientos, espíritu emprendedor, rasgos de personalidad, talentos, valores, visión empresarial

\section{Introducción}

El presente estudio tiene como propósito identificar los factores que inciden en los estudiantes de las Instituciones de Educación Superior (IES) para la generación de nuevos negocios (visión empresarial). Los factores como variables de estudio son: las habilidades, herramientas, conocimientos, valores, talentos y los rasgos de personalidad. Hoy en día la competitividad de los negocios en México está basada en quien emprende los negocios, es por ello que los factores que son relevantes para los emprendedores deben ser estudiados para tener una visión emprendedora que respalde los nuevos negocios. De acuerdo con la investigación de Hernández \& Arado (2015, pp 28-37), mencionan que el diseño de la visión nos permite plantear el futuro que deseamos alcanzar. De tal forma que definen la visión empresarial como los sueños en acción, que une los deseos, expectativas y las metas que deseamos lograr en nuestra vida, en los diferentes órdenes y esferas ubicados en al ámbito laboral. Por lo que se puede precisar el estado actual y proyectar el estado deseado en base a una serie de acciones para alcanzar 
su meta. La investigación de Dornelas (2002, pp. 299) menciona que el espíritu empresarial no es una moda, es consecuencia de los cambios, la tecnología y su velocidad combinada con competencia en la economía globalizada. La estructura del presente artículo está basada en el método científico, identificando el planteamiento del problema, el objetivo de la investigación, la hipótesis, el marco teórico, el método utilizado, el instrumento de medición, diseño y presentación de resultados.

\section{Planteamiento del problema}

En la actualidad los jóvenes estudiantes universitarios cuentan hoy en día con información y acceso a internet, que les facilita comercializar un producto y/o servicio, así como tener la opción de elegir la unidad de aprendizaje sobre actividades empresariales que les provee de una serie de acciones para que ellos emprendan un proyecto empresa; sin tener claro ¿Cuáles son los factores que favorecen el desarrollo de una visión empresarial a través del espíritu emprendedor?.

Por lo que resulta interesante explorar las variables independientes de estudio como son las habilidades, herramientas, conocimientos, valores, talentos y los rasgos de personalidad que contribuyen significativamente a descubrir su visión empresarial a través del espíritu emprendedor en el desarrollo de nuevos negocios en los estudiantes que cursan la unidad de aprendizaje del ámbito empresarial.

La razón última de profundizar en el tema de la visión empresarial no es otra que la de contribuir, conforme las aportaciones que arroja la presente investigación a los trabajos de estudio de otros autores inmersos en destacar el espíritu emprendedor dentro de una formación universitaria para proyección de estudiantes con un enfoque empresarial.

\section{Objetivo general de la investigación}

Demostrar que los estudiantes que cursan la unidad de aprendizaje del ámbito empresarial tienen visión empresarial a través del espíritu emprendedor para el desarrollo de nuevos negocios. 


\section{Hipótesis de investigación}

Los elementos que mayormente inciden en la visión empresarial a través del espíritu emprendedor son: las habilidades, las herramientas, los conocimientos, los valores, talentos, y los rasgos de personalidad en los estudiantes que cursan la unidade de aprendizaje del ámbito empresarial en la UANL, FACPYA.

a) A mayores habilidades del estudiante favorece el desarrollo de nuevos negocios a través del espíritu emprendedor.

b) El uso de las herramientas en clase presencial favorece el espíritu empresarial de los estudiantes.

c) Los conocimientos del estudiante son suficientes para el desarrollo de nuevos negocios.

d) Los valores que integran al estudiante favorecen el desarrollo de una visión empresarial.

e) Los talentos que posee el estudiante favorecen el desarrollo de una visión empresarial.

Los rasgos de personalidad del estudiante favorecen el desarrollo de una visión empresarial para el desarrollo de nuevos negocios.

\section{Marco teórico}

\section{La cultura empresarial}

"La competitividad de la nueva visión empresarial en México". Se puede apreciar en este artículo los retos que se vislumbran respecto a la nueva cultura empresarial de nuestro país, que se tornan difíciles, complejos y serios, pero no utópicos e inalcanzables. Por lo que este estudio concluye la importancia de identificar el tipo de desafíos que tenemos por delante y salir avante transformando nuestra filosofía como personas y empresarios proactivos, para contribuir a la transformación de nuestro país en cuatro áreas fundamentales: Educación, actitud ante la naturaleza, la religión y ante la vida misma. Haciendo referencia sobre las Instituciones de Educación Superior deben de estar preparadas para capacitar con calidad, desterrar una cultura identificada por preparar a incansables buscadores de empleo y, por 
el contrario, asumirse como generadoras vanguardistas de oportunidades para empresarios exitosos, columna vertebral de nuestras micro, pequeñas, medianas y grandes empresas Mexicanas. (Ramírez, 2005).

Por la importancia que tienen, diversos autores atribuyen la falta de competitividad a factores asociados al entorno macroeconómico, institucional, industrial, político y social, mientras que otros autores la atribuyen a las carencias del empresario (ausencia de cualidades propias del emprendedor, tales como tener objetivos claros, tomar decisiones sin temor al riesgo, ser visionario, organizado y planeador, entre otras cosas) y a su ineficiente gestión empresarial, de tal manera que es el emprendedor y sus habilidades las que en mayor medida determinan el éxito de la empresa. (Mendoza, 2014).

\section{La esencia del emprendedor}

El emprendedor es una figura producto de la sociedad, pero capaz de transformarla y dirigirla, son personas con cualidades para dirigir el desarrollo y crear organizaciones exitosas (Varela, 2001). Por otro lado, Gartner, (1985), propone considerar los elementos esenciales del estudio del emprendedor como son: el entorno, las características del individuo, el proceso y la organización creada.

En recientes estudios se hace notable la importancia que tiene el perfil psicológico del emprendedor como lo menciona el investigador McClellan (1961), destacando los atributos significativos en un emprendedor como son: la originalidad e innovación, tomar riesgos calculados, aceptación de sus responsabilidades, conocimientos de los resultados de sus actos, planificación en base al largo plazo. Por lo que también predomina en sus estudios en relacionar a los emprendedores atributos como es la necesidad de logro, autoconfianza, creatividad, la autonomía y el optimismo (David son, 1989; Boyadnos et al., 2000).

De acuerdo con el trabajo de (Fuentes \& Sánchez, 2010), sobre "Análisis del perfil emprendedor: una perspectiva de género". En este estudio, se ha llevado a cabo una investigación empírica sobre los perfiles de los potenciales emprendedores bajo una amplia muestra de estudiantes universitarios, cuya formación precisamente se percibe como un importante 
recurso a la hora de encaminar el futuro laboral hacia el autoempleo. Se ha confirmado lo indicado por Shane (2003) en cuanto a la influencia de factores demográficos como el sexo en la propensión a crear empresas.

Derivado de lo anterior resulta muy interesante la aportación de Binks \& Vale (1990), que identifican tres categorías de empresario emprendedor: 1) el empresario reactivo: es el que responde a las necesidades de los mercados; 2) el empresario causante del desarrollo económico por su papel de innovador, y 3) el empresario que aporta, mejorando procesos 0 productos.

\section{La visión empresarial}

En la literatura revisada de los autores Hernández \& Areno 2015, (pp 27837), la visión emprendedora se considera muy relevante en todos los ámbitos de la sociedad, es especialmente significativa entre los jóvenes, caracterizado por su creatividad, innovación, espíritu emprendedor y aventurero, menor temor al riesgo y mayor sensibilidad hacia los cambios tecnológicos, lo que los hacen mayores candidatos a llevar a cabo este tipo de proyectos.

El espíritu emprendedor y los valores que lo integran

Diversos han sido los estudios que se han realizado en materia del espíritu emprendedor enfocados a su visión empresarial, algunos de ellos a nivel internacional son los siguientes: "El fomento del espíritu emprendedor en la escuela: Nuevos retos para la educación del siglo XXl". En este artículo publicado en España se destaca la importancia de las cualidades personales, las habilidades sociales, así como las habilidades de dirección y que el espíritu emprendedor se relaciona con la capacidad para planificar, dirigir equipos de trabajo, negociar, tomar decisiones, desarrolla la iniciativa personal, la confianza en uno mismo, la creatividad, desarrollo de actitudes de cooperación y de trabajo en equipo.

Por lo que se refiere al espíritu emprendedor (o espíritu empresarial) se define fundamentalmente como una actitud: "el espíritu empresarial es la actitud y el proceso de crear una actividad económica combinando la asunción de riesgos, la creatividad y la innovación con una gestión sólida, en 
una organización nueva o en una ya existente". Comisión de las Comunidades Europeas (2003).

"La evolución del espíritu empresarial como campo del conocimiento. Hacia una visión sistemática y humanista". En este artículo se concluye que el campo del conocimiento del espíritu emprendedor es identificado en una etapa pre teórica, dada la falta de un modelo teórico central aceptado que logre ofrecer una comprensión del fenómeno y que retome tanto sus elementos centrales como las relaciones entre estos. Por lo que considera necesario integrar las dimensiones sociales y de contextualización en el tiempo para enriquecer el poder explicativo de los nuevos modelos del espíritu emprendedor (Pereira, 2007).

Por otra parte, los investigadores García, Álvarez \& Reyna (2007), hacen énfasis en las características que configuran el espíritu emprendedor por lo que integraron dos grupos de factores, uno que contiene las características esenciales en el espíritu emprendedor que son los factores generadores y otro que corresponde a los factores potenciadores que contienen las características que contribuyen a desarrollar el espíritu emprendedor lo cual está documentado a través de sus estudios. Definiendo tres niveles los para los factores generadores primer nivel: creatividad, Garravant (1994), fuerte voluntad, Garravant \& O'Cinneide, (1994) y Gibb 1988), vanidad, nivel alto de profesionalidad (buena gestión), Robinson (1991), Garravant \& O'Cinneide (1994), Scherer (1997), y Prat (1986). Destacando en el segundo nivel, mucha confianza en las personas cuando empezaron a desarrollar el proyecto, mejor selección de personas que trabajan en la empresa mediante una adecuada política de personal. En el tercer nivel. Buen carácter (amable y extrovertido) Filella (1997), y Roberts (1991) e Independencia, Hawkins \& Turla (1987), Sexton \& Bowman-Upton (1991), Gibb (1988), y Roberts (1991).

Por lo que se destacan estos tres niveles característicos del espíritu empresarial de índole intrapersonal, exceptuando buena gestión y política de personal. En relación a los factores potenciadores, se destacan tres niveles: primer nivel ambición, buscar personas adecuadas a su equipo, Casson (1982). Espíritu de sacrificio, arriesgado, Clouse (1990), Stearn \& Hill (1996). El segundo nivel está integrado por la insatisfacción laboral Dovrev \& Barnett (1999). Necesidad, Scherer (1987) y Roberts (1991). Audacia, Scherer, Brodzinski \& Wiebe (1991). Tercer nivel lo integra el entusiasmo e ilusión. 
Por lo que los investigadores concluyen que, a los factores potenciadores, no le otorgamos la importancia que en la actualidad se le ha considerado en muchos trabajos. Por considerar que son características impulsoras, pero no determinantes. García, Álvarez \& Reyna, (2007).

En la publicación realizada por la DG de Política de la PYME y Secretaría General 2003, (pp 1-99) cuyo tema es El espíritu emprendedor el motor del futuro describe nueve valores indispensables del espíritu emprendedor como son: a) Personales, que a través del análisis e investigación se genere creatividad con nuevas ideas y proyectos; b)la Autonomía, actuar por sí mismo para la toma de decisiones e iniciativas; c) Confianza, creer en sus propias actitudes positivas, con sus propios recursos y lo que puede realizar; d) Tenacidad, nunca dejar lo que se emprende, preservar y actuar por lo que se quiere; e) Sentido de la responsabilidad, cumplir con lo que se obliga consigo mismo y con el grupo; f) Capacidad para asumir riesgos, actuar decididos ante situaciones difíciles; g) Liderazgo, ante la realización de proyectos, influir en los demás a través de sus habilidades, conocimientos y cualidades; h) Espíritu de equipo, trabajar con los demás en función de los objetivos y métodos de trabajo; i)Solidaridad, trabajar ante la aceptación de lo que el grupo u organización decida.

Características y actitudes del emprendedor

Reciente artículo sobre "La actividad empresarial y el perfil femenino" Surdes \& Aguilar (2013), destacan el perfil de características sociodemográficas y de actitudes del comportamiento emprendedor de mujeres empresarias de pequeñas empresas, resaltando las actitudes del comportamiento emprendedor en este orden: Autodisciplina, Trabajo arduo, Capacidad de negociación, Liderazgo, Autoconfianza, Creatividad, Intuición, Necesidad de realización y Propensión al riesgo. Destacando en el estudio la iniciativa, la creatividad y la autoconfianza en las mujeres como aspectos asociados al emprendimiento, en cambio en los varones, se relaciona con características como el deseo de enfrentarse a nuevos retos o el entusiasmo ante los proyectos.

En su trabajo de investigación Carrera (2012) enfocado en los factores que inciden en el desarrollo de las PyMES del sector manufacturero del Área Metropolitana de Monterrey, define que los factores personales del 
empresario inciden significativamente con un $23.9 \%$ en el desarrollo de la empresa los cuales se presentan a continuación: Personalidad, Actitud, Conocimientos y Capacidad Administrativa.

De acuerdo con el estudio desarrollado por Herrera \& Burgoa (2013), enfatizan en la importancia que deben contar los estudiantes con habilidades y actitudes que demandan las unidades de aprendizaje empresarial, en las instituciones educativas para alcanzar su visión emprendedora. Por lo antes expuesto se puede inferir que los diferentes estudios relativos al espíritu emprendedor están enfocados a la personalidad del emprendedor y la visión empresarial como detonante de una cultura institucional para el desarrollo económico de una región.

\section{Método}

El estudio es de tipo descriptivo; no experimental transversal, con enfoque cuantitativo, utilizando la encuesta como instrumento de medición. Se aplicó a estudiantes que cursan la unidad de aprendizaje "El Emprender Competitivo". El período de investigación se realizó en el año 2013-2014. La muestra estuvo constituida por 103 estudiantes de la Facultad de Contaduría Pública y Administración de la UANL.

Instrumento de investigación

Se utilizó un cuestionario estructurado en dos partes. La primera parte mide la variable de datos generales de los estudiantes, con los indicadores edad, género, carrera, escolaridad, estado civil, semestre. La segunda mide la variable de visión empresarial a través de su espíritu emprendedor enfocada en los siguientes indicadores:

- Habilidades

- Herramientas

- Conocimientos

- Valores

- Talentos

- Rasgos de la personalidad 
Diseño de la Investigación

Mediante el presente estudio no experimental transversal, se diseñó el instrumento de medición el cual se validó con alfas de Cronbach, se validó la colinealidad de variables y se corrió la regresión lineal con la finalidad de dar respuesta a las preguntas de investigación y conocer qué variables intervienen significativamente en la visión empresarial a través de factores del espíritu emprendedor de los estudiantes de la UANL, FACPYA para el desarrollo de nuevos negocios.

\section{Presentación de resultados}

Análisis de Alfa de Cronbach

Los grados de correlación que se tienen entre los ítems de cada uno de los constructos, la validez y confiabilidad del instrumento de medición considerando las variables independientes y dependiente muestran (véase tabla 1) un análisis del alfa de cronbach satisfactoria, por encima de 0.7 (Cronbach, 1951). De acuerdo a estos resultados las preguntas de cada uno de constructos no están correlacionadas, son totalmente independientes una de la otra, y que una no depende de la otra. Por lo tanto, no hay necesidad de eliminar ninguna pregunta.

Tabla 1. Alfa de Cronbach para cada una de las variables

$$
X 1, X 2, X 3, X 4, X 5, Y \text {. }
$$

\begin{tabular}{ccc}
\hline \multicolumn{1}{c}{ Variables } & Items originales & Alfa de Cronbach \\
\hline Variables Independientes & - & - \\
X1 = Habilidades & 10 & 0.784 \\
X2 = Herramientas & 7 & 0.689 \\
X3 = Conocimiento & 3 & 0.610 \\
X4 = Valores & 10 & 0.789 \\
X5 = Talentos & 7 & 0.792 \\
X6 = Rasgos de personalidad & 11 & 0.869 \\
Variable Dependiente & - & - \\
Y = Visión Emprendedora & 7 & 0.736 \\
\hline
\end{tabular}

Fuente: Edición propia. 
Generación de variables del modelo

Se generaron las variables independientes y la variable dependiente del modelo a través de promedios ponderados con el fin de realizar el análisis de la regresión lineal múltiple.

Regresión lineal múltiple

Las variables independientes $\left(X_{1}, X_{2}, X_{3}, X_{4}, X_{5}, X_{6}\right)$ se generaron con la muestra de 103 encuestas con respecto a la variable dependiente $(Y)$ con el objetivo de obtener la regresión lineal múltiple. Dichos valores son introducidos al software estadístico SPSS-V17. Una vez introducidos al software estadístico, podemos observar la linealidad (véase Figura 1) de acuerdo a Schwab (2007), el cual indica que correlaciones altas (>0.50) entre las variables es indicativo de linealidad (véase Tabla 2), nos asegura que la relación entre la variable dependiente ( $Y=$ VISIÓN EMPRENDEDORA) y las variables independientes $\left(X_{1}, X_{2}, X_{3}, X_{4}, X_{5}, X_{6}\right)$ es significativo a través del rango de todos los valores.

Figura 1. Linealidad de los datos, gráfica P-P de los residuales estandarizados de la regresión

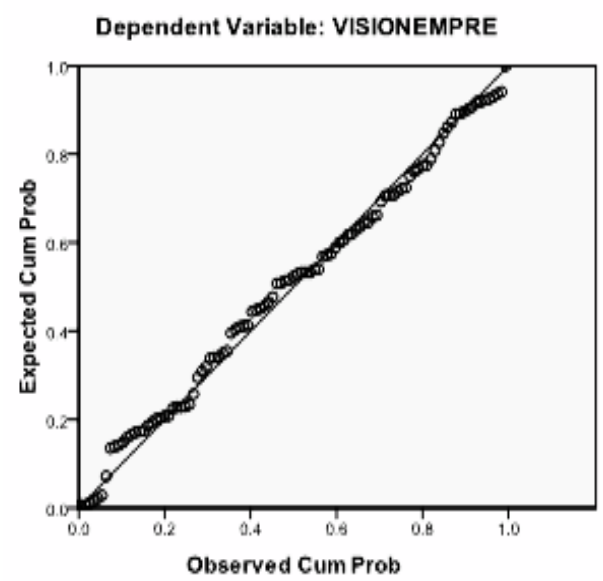

Fuente: SPSS-V17. 
Tabla 2. Matriz de correlaciones Inter-item

\begin{tabular}{|c|c|c|c|c|c|c|c|}
\hline Variable & Y & $X_{1}$ & $X_{2}$ & $X_{3}$ & $\mathrm{X}_{4}$ & $X_{5}$ & $X_{6}$ \\
\hline Correlación de Pearson & - & - & - & - & - & - & - \\
\hline$Y$ & 1.000 & .444 & .288 & .006 & .501 & .487 & .589 \\
\hline$X_{1}$ & .444 & 1.000 & .511 & .159 & .645 & .578 & .529 \\
\hline$X_{2}$ & .288 & .511 & 1.000 & .230 & .463 & .339 & .500 \\
\hline$X_{3}$ & .006 & .159 & .230 & 1.00 & .171 & .141 & .163 \\
\hline$X_{4}$ & .501 & .646 & .463 & .171 & 1.000 & .506 & .651 \\
\hline$X_{5}$ & .487 & .576 & .338 & .141 & .606 & 1.000 & .743 \\
\hline$X_{6}$ & .569 & .629 & .500 & .163 & .651 & .743 & 1.000 \\
\hline Sig. (1tailed) & - & - & - & - & - & - & - \\
\hline$Y$ & & .000 & .002 & .474 & .000 & .000 & .000 \\
\hline$X_{1}$ & .000 & & .000 & .054 & .000 & .000 & .000 \\
\hline$X_{2}$ & .002 & ,000 & & .010 & .000 & .000 & .000 \\
\hline$x_{3}$ & .474 & 054 & .010 & & .042 & .077 & .050 \\
\hline$X_{4}$ & .000 & .000 & .000 & .042 & & .000 & .000 \\
\hline$X_{5}$ & .000 & .000 & .000 & .077 & .000 & & .000 \\
\hline$X_{6}$ & .000 & .000 & .000 & .050 & .000 & .000 & \\
\hline $\mathrm{N}$ & - & - & - & - & - & - & - \\
\hline$Y$ & 103 & 103 & 103 & 103 & 103 & 103 & 103 \\
\hline$X_{1}$ & 103 & 103 & 103 & 103 & 103 & 103 & 103 \\
\hline$X_{2}$ & 103 & 103 & 103 & 103 & 103 & 103 & 103 \\
\hline$X_{3}$ & 103 & 103 & 103 & 103 & 103 & 103 & 103 \\
\hline$X_{4}$ & 103 & 103 & 103 & 103 & 103 & 103 & 103 \\
\hline$X_{5}$ & 103 & 103 & 103 & 103 & 103 & 103 & 103 \\
\hline$X_{6}$ & 103 & 103 & 103 & 103 & 103 & 103 & 103 \\
\hline
\end{tabular}

Notas: $Y=$ VISIÓN EMPRENDEDORA, $X_{1}=$ HABILIDADES, $X_{2}=$ HERRAMIENTAS, $X_{3}=$ CONOCIMIENTO, $X_{4}=$ VALORES,$X_{5}=$ TALENTOS, $X_{6}=$ RASGOS DE PERSONALIDAD

Fuente: SPSS-V17

Los valores de los coeficientes de correlación en las ciencias sociales, debajo de 0.20 no tienen utilidad práctica, entre 0.20 y 0.49 tiene utilidad práctica y entre 0.50 y 0.70 tiene una muy alta utilidad práctica (Cashin, 1988). 
Para probar la normalidad de los datos se aplica la prueba Kolmogorov-Smirnov, las medias y desviaciones estándares salen positivas como se muestra en la tabla 3. La normalidad de los datos entre las variables dependientes " $Y$ " debería estar normalmente distribuida con cada combinación de valores de las variables independientes $\left(X_{1}, X_{2}, X_{3}, X_{4}, X_{5}\right.$, $X_{6}$ ), (véase Figura 2).

Figura 2. Histograma y normalidad de los datos Dependent Variable: VISIONEMPRE

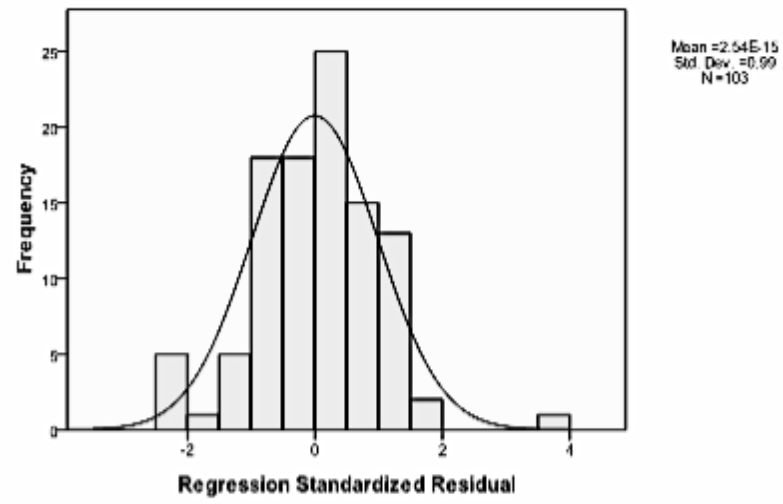

Tabla 3. Medias y desviaciones estándar de $Y, X_{1}, X_{2}, X_{3}, X_{4}, X_{5}, X_{6}$

\begin{tabular}{lccc}
\hline \multicolumn{1}{c}{ Variable } & Media & Desviación Estándar & $\mathbf{N}$ \\
\hline VISION EMPRESARIAL & 4.0965 & .57010 & 103 \\
HABILIDADES & 4.0096 & .53826 & 103 \\
HERRAMIENTAS & 3.5435 & .61301 & 103 \\
CONOCIMIENTO & 2.3731 & 1.17144 & 103 \\
VALORES & 4.2000 & .55186 & 103 \\
TALENTOS & 4.0997 & .58559 & 103 \\
RASGOS DE PERSONALIDAD & 4.1931 & .52835 & 103 \\
\hline
\end{tabular}

Fuente: SPSS-V17.

Al ejecutar la regresión lineal de las variables independientes, $X_{1}=$ HABILIDADES,$\quad X_{2}=$ HERRAMIENTAS,$\quad X_{3}=$ CONOCIMIENTO, $X_{4}=$ VALORES, $X_{5}=$ TALENTOS, $X_{6}=$ RASGOS DE PERSONALIDAD con respecto a la variable dependiente $Y=$ VISIÓN EMPRESARIAL se obtuvieron los siguientes resultados. 
Para comprobar la presencia de auto correlación, Durbin-Watson comprueba la independencia de residuales, arrojando un valor de 2.034 asegurando que no existe auto correlación en el modelo mostrando una regresión lineal valida (rango aceptado entre 1.5 a 2.5), con coeficiente de correlación aceptada (r) de $59.4 \%$, al igual que el coeficiente de determinación $\left(\mathrm{r}^{2}\right)$ de $35.3 \%$ para el modelo 2 (véase Tabla 4).

Tabla 4. Resultados de la regresión lineal con $Y^{c}$

\begin{tabular}{ccccccccccc}
\hline Model & $\mathbf{R}$ & $\begin{array}{c}\mathbf{R} \\
\text { Square }\end{array}$ & $\begin{array}{c}\text { Adjusted } \\
\mathbf{R} \text { Square }\end{array}$ & $\begin{array}{c}\text { Std. Error of } \\
\text { the Estimate }\end{array}$ & $\begin{array}{c}\text { R Square } \\
\text { Change }\end{array}$ & $\begin{array}{c}\mathbf{F} \\
\text { Change }\end{array}$ & df1 & df2 & $\begin{array}{c}\text { Sig. F } \\
\text { Change }\end{array}$ & $\begin{array}{c}\text { Durbin- } \\
\text { Watson }\end{array}$ \\
\hline 1 & $.569 \mathrm{a}$ & .323 & .317 & .47128 & .323 & 48.264 & 1 & 101 & .000 & \\
2 & $.594^{\mathrm{b}}$ & .353 & .340 & .46320 & .029 & 4.552 & 1 & 100 & .035 & 2.034 \\
\hline
\end{tabular}

Notas: a. Predictors: (Constant), RASGOSPERS

b. Predictors: (Constant), RASGOSPERS, VALORES

c. Dependent Variable: VISIONEMPRE

Fuente: SPSS-V17.

La multicolinealidad de las variables en los dos modelos es aceptable a través de la Durbin-Watson con 2.034. La explicación del modelo se da en un $32.3 \%$ para el modelo 1 , mientras que para el modelo 2 en un $35.3 \%$.

Para determinar el modelo que refleja significancia entre las variables podemos observar que no se muestra multicolinealidad entre las variables independientes mostrando una colinealidad aceptables mediante el índice de factor de inflación de varianza (VIF) debido a que son menores a 10 (véase Tabla 5).

Tabla 5. Índice de factor de inflación de varianza para $Y$

\begin{tabular}{|c|c|c|c|c|c|c|c|c|c|c|c|c|}
\hline \multirow[b]{2}{*}{ Model } & \multicolumn{3}{|c|}{$\begin{array}{l}\text { Unstandardized Standardized } \\
\text { Coefficients Coefficients }\end{array}$} & \multirow[b]{2}{*}{$\mathrm{t}$} & \multirow[b]{2}{*}{ Sign. } & \multicolumn{2}{|c|}{$\begin{array}{l}95 \% \text { Confidence } \\
\text { Interval for B }\end{array}$} & \multicolumn{3}{|c|}{ Correlations } & \multicolumn{2}{|c|}{ Collinearity Statistics } \\
\hline & B & $\begin{array}{l}\text { Std. } \\
\text { Err. }\end{array}$ & Beta & & & $\begin{array}{l}\text { Lower } \\
\text { Bound }\end{array}$ & $\begin{array}{l}\text { Upper } \\
\text { Bound }\end{array}$ & $\begin{array}{l}\text { Zero- } \\
\text { order }\end{array}$ & Partial & Part & Tolerance & VIF \\
\hline 1 (Constant) & 1.524 & .373 & & 4.082 & .000 & .783 & 2.264 & & & & & \\
\hline RASGOSPERS & 614 & .088 & .569 & 6.947 & .000 & .438 & .789 & .569 & .569 & .569 & 1.000 & 1.000 \\
\hline 2 (Constant) & 1.209 & .395 & & 3.057 & .003 & .424 & 1.993 & & & & & \\
\hline RASGOSPERS & .455 & .114 & .421 & 3.974 & .000 & .228 & .682 & .569 & .369 & .320 & .576 & 1.737 \\
\hline VALORES & .234 & .110 & .226 & 2.133 & .035 & .016 & .451 & .501 & .209 & .172 & .576 & 1.737 \\
\hline
\end{tabular}

Notas: Dependent Variable: VISIONEMPRE

Fuente: SPSS-V17. 


\section{Análisis de varianza}

El análisis de varianza ANOVA compara las medias de cada variable para demostrar que existe independencia entre las variables independientes, por lo que se deduce que el modelo de la tabla 6 muestra independencia contra las otras variables por tener una significancia por debajo del $5 \%$.

Tabla 6. Análisis de Varianza ANOVA para Yc

\begin{tabular}{lccccc}
\hline \multicolumn{1}{c}{ Model } & Sum of Squares & df & Mean Square & F & Sig. \\
\hline 1 Regression & 10.720 & 1 & 10.720 & 48.264 & $.000^{\mathrm{a}}$ \\
Residual & 22.432 & 101 & .222 & & \\
Total & 33.152 & 102 & & & \\
& & & & & \\
2 Regression & 11.696 & 2 & 5.848 & 27.256 & $.000^{\mathrm{b}}$ \\
Residual & 21.456 & 100 & .215 & & \\
Total & 33.152 & 102 & & & \\
\hline
\end{tabular}

Notas: a. Predictors: (Constant), RASGOSPERS

b. Predictors: (Constant), RASGOSPERS, VALORES

c. Dependent Variable: VISIONEMPRE

Fuente: SPSS-V17.

\section{t-student}

De acuerdo a las significancias de la t-student considerando (véase tabla 5) un error estimado menor al $5 \%$, podemos concluir que las variables que impacta significativamente a la VISIÓN EMPRENDEDORA en los emprendedores son la $\mathrm{X}_{4}=$ VALORES, por tener un valor de significancia del 0.035 , la $X_{6}=$ RASGOS DE PERSONALIDAD con valor de significancia del .000 . La ecuación lineal de la presente investigación para la variable dependiente $\mathrm{Y}=$ VISIÓN EMPRENDEDORA de los emprendedores quedaría de la siguiente manera.

$$
\hat{Y}=1.209+0.455 X_{6}+0.234 X_{4}
$$

Las variables excluidas para la variable dependiente $Y=$ VISIÓN EMPRENDEDORA, fueron: $X_{1}=$ HABILIDADES, $X_{2}=$ HERRAMIENTAS, $X_{3}=$ CONOCIMIENTO, $X_{5}=$ TALENTOS, esto derivado de las significancias que obtuvieron con la t-student, un error estimado arriba del $5 \%$ por lo que se deduce que esta variable no es confiable para el modelo. 


\section{Conclusiones}

En el presente estudio se aplicaron 103 encuestas a estudiantes de la FACPYA de la UANL, los valores de alfa de Crombach fueron satisfactorias para una de las variables, el índice de factor de inflación de varianza (VIF) nos indica que no hay multicolinealidad entre variables lo cual, valida el modelo propuesto, se obtuvieron indicadores de regresión significativas (t) por lo cual la encuesta se puede utilizar para futuros estudios.

El desarrollo del presente estudio, permitió que se respondiera a la hipótesis de investigación; los elementos que mayormente inciden en la visión empresarial a través de su espíritu emprendedor son: los valores, y los rasgos de personalidad en los estudiantes que cursan las unidades de aprendizaje del ámbito empresarial en la UANL, FACPYA. Con un 45.5\% impactan los rasgos de personalidad en la visión emprendedora, mientras en un $23.4 \%$ impactan los valores en la visión emprendedora para la generación de nuevos negocios.

\section{Discusión de resultados}

En la actualidad en pleno siglo XXI el papel que juega el emprendedor es de vital importancia por lo que se propone que en las IES, Bygrave \& Hofer (1991), proponen que se amplíen sus programas de estudio en el desarrollo del espíritu emprendedor y sus características personales y las funcionales del emprendedor, que incluya el estudio del proceso emprendedor y sus características. De tal forma que el trabajo de Pereira (2007) describe que se realice un conjunto de funciones, actividades y acciones asociadas con percepción de oportunidad y la creación de la organización para explotarla.

Los trabajos realizados por la OCDE, (1998). En el tema del espíritu y las iniciativas emprendedoras en una determinada economía mencionan que son el resultado de factores procedentes de tres dimensiones primordiales en las que se destacan: el entorno macroeconómico e institucional, programas gubernamentales y el marco cultural.

La aportación del presente estudio es implementar estrategias en las instituciones educativas donde se consideren los factores significativos de esta investigación como son: los valores y los rasgos de personalidad en el estudiante, para generar una visión emprendedora, en los valores se deben 
desarrollar la creatividad, autonomía, confianza en sí mismo, tenacidad, sentido de responsabilidad, capacidad para asumir riesgos, liderazgo, espíritu de equipo, solidaridad y respeto, y con respecto a los rasgos de personalidad, se deben tener un alto nivel de energía y entusiasmo para emprender, comportarse con calma en situaciones de ambigüedad 0 inciertas, aceptar desafíos y tomar riesgos calculados, trabajar lo suficiente para dar resultados, contar con ideas de tal manera de hacer diferente las cosas, tener buena relación con los compañeros de trabajo, supervisar los trabajos de los demás, obtener retroalimentación de su trabajo, establecer sus propios estándares y colocarse en situaciones donde tome iniciativa.

Es importante indagar sobre las variables que no fueron significativas, las cuales son: las habilidades, las herramientas, los conocimientos y los talentos, con el fin de profundizar en las variables de estudio, así como en los diversos enfoques que contribuyen al espíritu emprendedor como son: la libertad, la educación, el entorno y un fortalecido escenario institucional, citado en Gutiérrez \& Amador (2011).

Este trabajo de investigación reafirma el estudio realizado por Carrera (2012) cuya aportación del estudio fue la variable independiente rasgos de personalidad que contribuyen significativamente en el desarrollo empresarial como lo son: Personalidad, Actitud, Conocimientos y Capacidad Administrativa. De tal manera que el estudio realizado por Herrera \& Burgoa (2013) aporta la importancia de que los estudiantes deben contar con habilidades y actitudes que se demandan en las unidades de aprendizaje en las IES para alcanzar su visión emprendedora.

\section{Referencias}

Binks, M. \& Vale, P. (1990). Entrepreneurship and economic change. Londres: McGraw-Hill. Carrera, M. (2012). Factores que intervienen en el desarrollo de las PyMES del sector manufacturero en cinco municipios del Área Metropolitana de Monterrey, afiliadas a la CAINTRA. Tesis doctoral, San Nicolás de los Garza: Universidad Autónoma de Nuevo León.

Comisión de las Comunidades Europeas (2003). Libro verde. El espíritu empresarial en Europa. Bruselas: Comisión de las Comunidades Europeas.

Davidsson, P. (1989): Continued entrepreneurship and small firm business. Stockholm: Stockholm School of Economics. 
Fuentes, F. \& Sánchez, S. (2010). Análisis del perfil emprendedor: Una perspectiva de género, Estudios de Economía Aplicada, 28(3), 1-28.

García, J., Álvarez, P. \& Reyna, R. (2007). Características del emprendedor de éxito en la creación de PYMES españolas, Estudios de Economía Aplicada, 25(3), 951-974

Gartner, W. B. (1985). A conceptual framework for describing the phenomenon of new venture creation, Academy of Management Review, 10(4), 696-706.

Gutiérrez, A. M. \& Amador M. E. (2011). El potencial emprendedor en los estudiantes de la carrera de contabilidad de las universidades San Marcos Perú y Guadalajara de México- Centros Universitarios de los Altos- Análisis comparativo. QUIPUKAMAYOC Revista de la Facultad de Ciencias Contables, 19(36), 28-37.

Herrera, E. \& Burgoa, T. (2013). La importancia del perfil vocacional en la visión del estudiante universitario: Caso de estudio en facultades de la Universidad Autónoma de Nuevo León, UANL, International Journal of Good Conscience, 8(2) 23-47.

Hernández, C. \& Arano, R. (2012). El desarrollo de la cultura emprendedora en estudiantes universitarios, Ciencia Administrativa, 1, 28-37.

McClelland, D. C. (1961). The achieving society. Princeton: D. Van Norstrand.

Mendoza, S. A. (2014); Supervivencia de la micro, pequeña y mediana empresa (MIPYME), Escuela de Negocios: Revista CESUN Universidad, 1(1), 36-48.

Pereira, F. (2007). La evolución del espíritu empresarial como campo del conocimiento. Hacia una visión sistemática y humanista, Cuadernos de Administración, 20(34), 11-37.

Ramírez, L. (2005). La competitividad de la nueva visión empresarial en México, Episteme, $1(4), 28-37$

Dornelas, J. (2002). Planeando incubadoras de empresas: como desenvolver un plano de negocios para incubadoras. Rio de Janeiro: Futura.

OCDE (1998). Fostering entrepreneurship. Paris: OCDE.

Shane, S. (2003). A general theory of entrepreneurship: The individual-opportunity nexus. Cheltenham: Edward Elgar.

Surdes, E., Aguilar, N. (2013). La actividad empresarial y el perfil femenino: Mujeres, su participación económica en la sociedad. Cd. México. Editorial Universitaria.

Varela, R. (2001). Innovación empresarial, arte y ciencia en la creación de empresas. 2da edición, Bogotá: Pearson educación de Colombia. 